\title{
Taxa de Desemprego Não Aceleradora da Inflação: uma estimação da NAIRU para a economia brasileira no período de 2000 até 2013
}

Non Accelerating Inflation Rate of Unemployment (NAIRU): an estimation for the Brazilian economy between 2000 and 2013

\author{
Luma de Oliveira \\ Universidade Federal do Rio Grande do Sul \\ Marcelo Savino Portugal \\ Universidade Federal do Rio Grande do Sul \\ Mateus Boldrine Abrita \\ Universidade Federal do Rio Grande do Sul
}

\begin{abstract}
In this paper are we develop estimates for the Non-Accelerating Inflation Rate of Unemployment (NAIRU) for the Brazilian economy. The data used are quarterly for the period between 2000Q1 and 2013Q2. For the unemployment rate we used DIEESE's data, and for the inflation rate data from IBGE (National Consumer Price Index INPC). The model was estimated based on a transfer equation. The central results are that there was a shift in the correlation coefficient between unemployment and inflation, from positive to negative, while the NAIRU remained above the unemployment rate on the period analysed.
\end{abstract}

\section{Keywords}

unemployment rate; inflation rate; correlation coefficient; NAIRU.

JEL Codes E; E3; E31.

\section{Resumo}

Neste artigo são desenvolvidas estimativas da Taxa de Desemprego Não Aceleradora da Inflação (NAIRU) para a economia brasileira. Esta é estimada com dados trimestrais de 2000 ao segundo trimestre de 2013 da taxa de desemprego aberta no periodo de referência de 30 dias do DIEESE e da taxa de inflação pelo Índice Nacional de Preços ao Consumidor (INPC). A metodologia empregada condiz com uma equação de transferência. Os principais resultados dizem respeito à mudança do coeficiente de correlação entre a taxa de inflação e a taxa de desemprego, que passou de negativo para positivo e a permanência da taxa NAIRU acima da taxa de desemprego no periodo em questão.

\section{Palavras-chave}

taxa de desemprego; taxa de inflação; coeficiente de correlação; NAIRU.

Códigos JEL E; E3; E31. 


\section{Introdução}

Nos anos de 1990 com a dinâmica social e econômica imposta pela demanda da sociedade, ou seja, pela inserção obrigatória no processo de globalização da economia, o Brasil enfrentou diversas transições sociais, políticas, tecnológicas e econômicas que afetaram profundamente sua economia, seu mercado de trabalho e, consequentemente, o desemprego.

Com a implementação do Plano Real em 1994, alcançou-se a tão quista estabilização, porém, esta foi acompanhada principalmente pelo custo social do aumento do desemprego e pelo baixo dinamismo da economia brasileira. Assim, a taxa de desemprego que vinha em uma trajetória de crescimento desde o início do plano reverte a tendência em 2003, atingindo um pico, e passando a alcançar patamares menores aos de antes deste.

Neste contexto, remete-se ao conceito do trade-off desenvolvido inicialmente por Alban William Phillips em 1958 - a relação negativa entre a inflação e o desemprego. Conceito amplamente discutido por Friedman (1968) e Phelps (1968). Os autores argumentavam que se o governo tentasse deixar o desemprego mais baixo aceitando inflação mais alta, o dilema desapareceria, e a taxa de desemprego não poderia ser sustentada abaixo de um determinado nível que eles chamavam de taxa natural de desemprego.

Sob este prisma, foram adicionadas as expectativas de inflação à curva e a ideia de que é necessária uma taxa natural de desemprego para manter constante a taxa de inflação, ou seja, a taxa de desemprego não aceleradora da inflação ou NAIRU (da expressão em inglês "Non-Accelerating Inflation Rate of Unemployment"), abriu um leque de inúmeras tentativas de estimação desta taxa.

Desta forma, este artigo se concentra na metodologia empregada por Portugal e Madalozzo (2000), na qual os autores estimam a NAIRU com dados trimestrais do início dos anos 1980 até meados da década de 1990, a partir de uma equação de transferência. A variável endógena (no caso a diferença entre a inflação corrente e a esperada) se comporta de acordo com as influências de seus valores passados e correntes dela própria e da variável exógena.

Assim, o artigo, além desta introdução se desenvolve em mais três seções. A segunda seção faz uma pequena revisão do panorama da economia brasileira pós-implementação do Plano Real, seguida de uma revisão empírica de trabalhos que já calcularam a taxa NAIRU. Posteriormente, a próxi- 
ma revela os passos para a estimação da NAIRU deste trabalho - o primeiro subitem faz uma análise dos dados utilizados e a metodologia empregada; e, no segundo, os resultados empíricos da estimação. Por fim, apresenta-se as considerações finais, as referências bibliográficas e dois anexos.

\section{Revisão de fatos estilizados e empírica}

Nesta seção será discutido o panorama da economia brasileira, que levou a escolha do período a ser analisado por este artigo, além de apresentar uma revisão empírica sobre trabalhos que já estimaram a taxa NAIRU para o Brasil e para outras economias.

\subsection{Fatos estilizados}

Nos anos noventa do século XX, dado um movimento internacional de maior abertura econômica (Consenso de Washington) e com a dinâmica social e econômica imposta pelas demandas da própria sociedade, o Brasil enfrentou diversas transições sociais, culturais, políticas, tecnológicas e econômicas, fruto de um processo modernizante de inserção na economia mundial globalizada que afetaram profundamente sua economia, seu mercado de trabalho e, consequentemente, o desemprego. A abertura comercial e financeira no início da década de 1990 foi uma delas, pois, ao mesmo tempo em que o coeficiente de abertura comercial ${ }^{1}$ da economia brasileira era pequeno e começava a aumentar, o mercado de trabalho passou por desafios maiores como o caso de que o avanço tecnológico é um poupador de mão de obra (Pichetti; Chahad, 2003).

A economia brasileira, ao longo da década de 1980 e 1990, lançou inúmeros planos de estabilização inflacionária que não obtiveram resultados satisfatórios em relação aos seus objetivos, como os Planos Cruzado, Bresser, Verão e Collor. Entretanto, com o Plano Real em 1994, o tão almejado controle do nível de preços foi alcançado de forma eficiente. A principal diferença em relação aos outros planos de estabilização foi o fato de que o país estava inserido no fluxo voluntário de recursos externos e de bens.

1 Calculado pela soma das exportações e importações relativamente ao PIB nacional. 
Sendo assim, seria mais difícil que os agentes econômicos internos, expostos à concorrência dos produtos internacionais, repassassem possíveis choques positivos para os preços dos seus produtos que afetavam a economia, ou seja, a reserva de mercado foi diminuída. Um volume significativo de reservas havia se acumulado, o plano não foi implementado de surpresa e não houve congelamentos, mas sim uma substituição natural da moeda (Gremaud et al., 2009).

Segundo Flori (2003), as consequências da estabilização se deram principalmente pelo custo social do aumento do desemprego e pelo baixo dinamismo da economia brasileira, isto porque, as melhorias do quadro econômico social, ocorridas com o Plano de Estabilização, não produziram a ampliação esperada das oportunidades ocupacionais.

Nos primeiros anos de sua implantação, ocorreu um desgaste da âncora cambial como instrumento básico da política econômica, pois, de um lado havia uma deterioração da conta corrente, e do outro, havia a necessidade de compensar o déficit mediante entrada de capital. Deste modo, os agentes se sentiam atraídos pela alta taxa de juros, o que gerava despesas financeiras significativas e piorava a relação dívida pública/PIB (Giambiagi et al., 2005).

Desta forma, o Brasil adotou, em 1o de julho de 1999, o Regime de Metas de Inflação (RMI), após um período turbulento de crise de confiança nos mercados emergentes. Dado que havia se tornado insustentável a manutenção da âncora cambial, tendo em vista os ataques especulativos e baixos níveis nas reservas internacionais. O câmbio passou a flutuar em janeiro do mesmo ano e o regime de metas foi inserido como âncora nominal.

De acordo com Mendonça (2001), ao analisar os mecanismos de transmissão da política monetária brasileira, após 1994 e no período do regime de metas para inflação, o instrumento dos juros adquire papel central no combate à inflação. Deste modo, a taxa básica de juros seria a referência para as demais taxas de juros praticadas na economia e, assim, interferiria no setor real.

Segundo o autor uma política monetária que objetiva contrair a demanda agregada se dá da seguinte forma: a autoridade monetária adota uma política monetária contracionista gerando uma elevação da taxa de juros de curtíssimo prazo; considerando rigidez nos preços no curto prazo, a taxa de juros real se eleva, deixando o retorno real dos juros maior que a eficiência marginal do capital, pelo menos em parte da economia, assim, o nível de investimento cai seguido da demanda agregada e do nível de produto. 
Em outras palavras, o mercado de reservas bancárias está relacionado com a taxa básica de juros, influenciando o mercado financeiro, que por sua vez, determina o nível de empréstimos, poupança e financiamentos.

Neste contexto, o regime de metas de inflação brasileiro, na década de 2000, é caracterizado pelo controle do nível de preços via elevação na taxa de juro, através dos canais de transmissão da política monetária, ${ }^{2}$ que afetam a demanda agregada e os preços. Bresser-Pereira e Gomes (2009) argumentam que passados seis messes da adoção da flutuação cambial no Brasil, a estrutura do sistema de metas de inflação foi colocada em prática pelo Banco Central, estabelecendo metas, utilizando o IPCA como índice de referência e a taxa Selic, escolhida como instrumento de política monetária e estabelecida uma margem de tolerância de dois pontos percentuais. Assim sendo, o controle inflacionário era o objetivo principal da autoridade monetária, sem considerar que a taxa básica de juros muito elevada também é um obstáculo para uma estabilização macroeconômica ampla, em termos de produto, emprego, vulnerabilidade e do próprio nível de preços.

Com a mudança da âncora cambial para a taxa de inflação, esta conseguiu se manter relativamente estável. A taxa de desemprego que vinha em uma trajetória de crescimento desde a implementação do Plano Real, reverte tendência e passa a alcançar patamares menores aos de antes do plano (Gráfico 1).

\section{Gráfico 1 Taxa de desemprego e tendência}

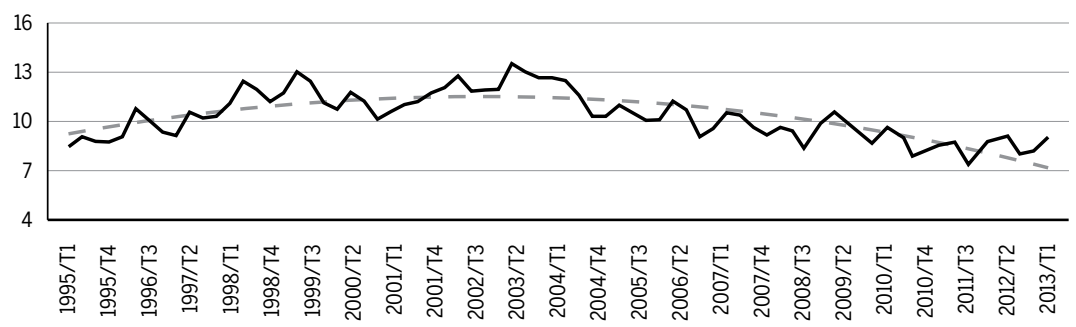

Trimestres

taxa de desemprego

polinômio (taxa de desemprego)

Fonte: Instituto Brasileiro de Geografia e Estatística (IBGE), elaboração própria.

2 Segundo Mendonça (2001) os principais mecanismos de transmissão da política monetária considerados pelo Comitê de Política Monetária do Banco Central do Brasil são: i) taxa de juros; ii) taxa de câmbio; iii) preço dos ativos; iv) crédito; e v) expectativas. Entretanto, o principal é o da taxa de juros. 
Neste contexto, remete-se ao conceito do trade-off desenvolvido inicialmente por Alban William Phillips em 1958, ou seja, a relação negativa entre a inflação e o desemprego. Isto porque, se o desemprego fosse alto, a inflação seria baixa, e, quando a inflação fosse alta, o desemprego seria baixo. Dessa forma, uma vez que instala a tendência de queda, a taxa de desemprego poderia ser a causa do processo inflacionário que a economia brasileira passou a enfrentar em anos recentes.

Então, se o governo tentasse deixar o desemprego mais baixo aceitando uma inflação mais alta, o dilema desapareceria e a taxa de desemprego não poderia ser sustentada abaixo de um determinado nível que Friedman (1968) e Phelps (1968) chamavam de taxa natural de desemprego. Sob este prisma, foram adicionadas as expectativas de inflação à curva, assim, ter-se-ia que a variação na taxa de inflação $\left(\pi_{t}-\pi^{e}\right)$ seria igual a diferença entre a taxa de desemprego no período atual $\left(U_{t}\right)$ e a taxa natural de desemprego $\left(U^{*}\right)$ multiplicado pelo negativo do coeficiente $(\beta)$, mais os resíduos $\left(\varepsilon_{t}\right)$. Ou seja,

$$
\pi_{t}-\pi^{e}=-\beta\left(U_{t}-U^{*}\right)+\varepsilon_{t}
$$

A taxa natural de desemprego é a taxa necessária para manter constante a taxa de inflação. É por isso que esta taxa é também chamada de Taxa de Desemprego Não Aceleradora da Inflação ou NAIRU (da expressão em inglês "Non-Accelerating Inflation Rate of Unemployment").

\subsection{Revisão empírica}

Antes de iniciar o processo de estimação a que este trabalho se dispõe, será feita uma revisão de alguns trabalhos que estimaram a NAIRU para o Brasil e para outros países.

Portugal e Madalozzo (2000) estimam a NAIRU com dados trimestrais do início dos anos 1980 até meados da década de 1990, destacando planos de estabilização que configuram o período nos anos 1986, 1989, 1990 e 1994. A primeira inovação do trabalho, dado que utilizam o Índice Nacional de Preços ao Consumidor (INPC) para representar a inflação e como taxa de desemprego as taxas abertas com período de referência de 30 dias da PME (IBGE) e PED (SEADE), é o fato da expec- 
tativa de inflação ser modelada como um processo autorregressivo de ordem um.

Além disso, para alcançar a NAIRU utilizam da equação de transferência. Isto porque a variável endógena (no caso a diferença entre a inflação corrente e a esperada) se comporta de acordo com as influências de seus valores passados e correntes dela própria e da variável exógena. Assim, tem-se,

$$
y_{t}=\alpha+A(L) y_{t-1}+C(L) z_{t}+B(L) \varepsilon_{t}
$$

Onde: $y_{t}=$ variável exógena no período $t$;

$z_{t}=$ sequência da variável exógena no período $t$;

$\varepsilon_{t}=$ resíduos da estimativa;

$A(L), B(L)$ e $C(L)=$ polinômios de lags.

Com o propósito de filtrar a série desemprego empregam o método de máxima verossimilhança para a estimação das variâncias dos resíduos de cada um dos componentes, sendo o vetor estimado a partir Filtro de Kalman.

Assim, alcançam a estimativa para a taxa NAIRU com o método de variáveis instrumentais a cada trimestre e comprovam sua veracidade por meio de uma estimava simples de mínimos quadrados da diferença desta taxa em relação a taxa de desemprego, contra a taxa de inflação. Assim, esta última estimação os faz alcançar um coeficiente consistentemente significativo e negativo.

Lima (2000) calcula a NAIRU para o Brasil e a investiga ao longo do tempo, assim os intervalos de confiança e sua utilidade na condução da política monetária no Brasil. $\mathrm{O}$ autor estima dois modelos, um com resíduos ARCH e outro com mudança de regime ]markoviana para dados trimestrais que vão de 1982 ao terceiro trimestre de 1999. Suas principais conclusões mostram que a NAIRU tem crescido desde 1995 e que existe correlação significativa e com sinal correto entre os desvios da taxa de desemprego em relação à taxa de inflação e à NAIRU. Acrescenta, porém, a suas conclusões que os intervalos de confiança das estimativas alcançadas são demasiadamente altos e que a NAIRU acaba sendo pouco útil na condução de política monetária.

Ball e Mankiw (2002) estimam a NAIRU para os Estados Unidos com dados anuais de 1960 a 2000. Primeiramente fazem a análise constante da taxa com base na seguinte equação: 


$$
\Delta \pi=a U^{*}-a U+v
$$

Alcançam um termo constante de 3,8 e o coeficiente de desemprego de $-0,63$, os quais geram uma taxa NAIRU de $6,1 \%$. Ressaltam o fato de que o cálculo dessa taxa constante é suscetível a muitas críticas, levando-os a calcular uma taxa que varia ao longo do tempo. Isso porque, na equação disposta acima o termo $(v)$ captura as flutuações de curto prazo e os movimentos em $\left(U^{*}\right)$ mudam ao longo tempo na relação inflação-desemprego. Dessa forma, manipulam a equação (2), ou seja,

$$
U^{*}+v / a=U+\Delta \pi / a
$$

O lado direito é computado dos dados e mede as mudanças na curva de Phillips o primeiro termo (quando não se considera a soma) representa a tendência de longo prazo e $v / a$ é a proporção dos choques de oferta de curto prazo. Dessa forma, alcançam $U^{*}$ através do filtro Hodrick Prescott que minimiza a soma dos quadrados dos resíduos entre a tendência e a série atual. Concluem destacando que a NAIRU, que pode ser vista como uma aproximação da taxa natural de desemprego, muito embora seja de fácil compreensão sua estimação não segue essa direção, ou seja, ela é de difícil medida e muda ao longo do tempo, além disso, destacam que a produtividade altera a relação inflação desemprego.

Ferreira et al. (2003) estimam a taxa NAIRU para o Brasil para o período de 1982 a 2002, segundo a abordagem de Ball-Mankiw, e comparam os resultados com outros trabalhos publicados. A NAIRU variou através do tempo, mas não muito durante o período de 1983/2001. A taxa de desemprego continuou abaixo da NAIRU de 1985/97, resultado este considerado consistente com as altas taxas de inflação observadas durante grande parte do período.

Silva Filho (2008) calculou a NAIRU para o Brasil no período de 1996 a 2006 com dados trimestrais e alcançou que a Curva de Phillips aceleracionista tem inclinação contrária ao que se esperaria teoricamente, ou seja, aumentos na inflação provocam um deslocamento no mesmo sentido para a taxa de desemprego. Desta forma, temos que a taxa de desemprego só foi menor que a NAIRU máxima $(8,5 \%)$ em poucos trimestres e, mesmo com o desemprego se distanciando da NAIRU em 2008 a inflação se desacelerou, quando pela teoria deveria acelerar. 
Sachsida, Ribeiro e Santos (2009) analisam três curvas de Phillips com mudança de regime para a economia brasileira no período de 1995-2008 com dados trimestrais, sendo que uma se refere ao estado do Rio de Janeiro, outra ao de São Paulo e uma geral para a economia brasileira. Utilizam como medida de inflação o IPCA e, como medida do custo marginal das empresas diferentes proxies para analisar, a partir de um modelo com mudança de regime markoviano, a possível não linearidade entre as variáveis.

O principal resultado diz respeito ao fato de que foi comprovada a não linearidade da Curva de Phillips para a economia brasileira e as estimativas que não a consideram podem estar viesadas. Como é o caso dos autores terem encontrado um papel maior para as expectativas de inflação ao invés da inflação esperada que habitualmente representa um peso maior nos modelos lineares, ou seja, estes podem estar subestimando o papel das expectativas.

\section{Estimação da NAIRU}

Esta seção tem o objetivo de apresentar a estimação a taxa de desemprego de equilíbrio para a economia brasileira no período de 2000 ao segundo trimestre de 2013. O período adotado consiste em uma amostra representativa e em linha com o propósito do trabalho, considerando a melhor utilização dos dados disponíveis. A primeira parte se refere à descrição dos dados e as diferentes metodologias empregadas. A segunda mostra os resultados alcançados nas estimações.

\subsection{Dados empíricos e metodologia econométrica}

A NAIRU será alcançada a partir estimação de uma Curva de Phillips, já conceituada na segunda seção. Para tanto, os dados utilizados serão a série Índice Nacional de Preços ao Consumidor (INPC) do IBGE, que mede a inflação registrada em 11 capitais, e a taxa de desemprego aberta ${ }^{3}$ com período de referência de 30 dias da PED (DIEESE/Fundação SEADE-SP). ${ }^{4}$

3 A taxa de desemprego aberto se refere as pessoas que procuraram trabalho de maneira efetiva nos 30 dias anteriores ao da entrevista e não exerceram nenhum trabalho nos sete últimos dias.

4 Muito embora o trabalho se baseie na metodologia empregada no artigo de Portugal e 
Vale ressaltar que a Pesquisa de Emprego e Desemprego (PED) é um levantamento domiciliar contínuo, realizado mensalmente. Iniciou-se na Região Metropolitana de São Paulo, em convênio entre o DIEESE e a Fundação Seade, e se alastrou para o Distrito Federal e as Regiões Metropolitanas de São Paulo, Porto Alegre, Belo Horizonte, Salvador, Recife e, mais recentemente, Fortaleza, constituindo o Sistema PED nos anos subsequentes (Sistema de Pesquisa de Emprego e Desemprego, 2014).

Para os dados da inflação esperada, muitas já foram as metodologias empregadas por diversos autores para alcançar essa série. Especificadamente neste trabalho, foi empregada a metodologia de modelos de séries temporais univariados (Modelos ARMA) a fim de determinar o processo estocástico gerador.

Os modelos ARMA compreendem uma classe de modelagem que combinam termos autorregressivos (AR) e/ou médias móveis (MA), sendo que MA consiste em uma combinação linear dos termos de erro ruído branco. Desta forma, atinge-se um modelo ARIMA (p, d, q), em que p é o número de termos autorregressivos, q é o número de termos de média móvel e d é a ordem de integração (Bueno, 2011).

Para o processo gerador ser estável é necessário que a série seja estacionária, pois, desta forma, é possível construir um modelo válido para estimação de valores futuros. Uma vez que a série a ser prevista, ou seja, o INPC, mostrou-se estacionária em nível, a identificação do processo gerador se dá pela análise das funções de autocorrelação (FAC) e autocorrelação parcial (FACP). ${ }^{5}$

Assim, a série que representa as expectativas de inflação a ser utilizada nas estimações do objetivo deste trabalho foi alcançada a partir de um processo autorregressivo de ordem um, estando em consonância com muitos autores.

Já se preocupando com o processo de suavização dos dados, foram utilizados dados trimestrais ${ }^{6}$ para as três séries adotadas para fins da estimação da NAIRU. Neste contexto, os dados requeridos serão para o período de 2000 ao segundo trimestre de 2013.

Madalozzo (2000) e os autores tenham utilizado a série disponibilizada pela Pesquisa Mensal do Emprego (PME), este artigo fará uso apenas dos dados do DIESSE pelo fato do período escolhido a ser estimado. Isto porque, assim como a PME que produz indicadores mensais sobre a força de trabalho que permitem avaliar as flutuações a tendência, a PED é estruturada da mesma forma e nos permitirá fazer as análises pretendidas.

5 O correlograma que dispõe as FAC e FACP é apresentado no Anexo I deste artigo, assim como as estimativas do processo estocástico gerador dos dados.

6 Uma vez que as séries disponibilizadas pelo IBGE e pelo DIEESE são mensais, foi necessária a compactação dos dados em trimestres. Para a série taxa de desemprego foi feita uma média aritmética simples (software Gret) já para a taxa de inflação utilizou-se de uma média geométrica. 
A metodologia empregada se baseia no modelo de função de transferência ${ }^{7}$ (equação 2) descrito no artigo de Portugal e Madalozzo (2000). Esse procedimento parte do pressuposto de que ambas as séries são estacionárias e que a variável explicativa seja exógena.

A variável endógena se dá pela diferença da taxa de inflação corrente e da expectativa de inflação, assim, mesmo que o INPC não se mostrasse estacionário em nível (o que não aconteceu), a diferença seria.

Para o tratamento da taxa de desemprego, ${ }^{8}$ foi utilizado o procedimento verificado no artigo de Ball e Mankiw (2002), ou seja, do filtro Hodrick Prescott (HP). ${ }^{9}$ Esta é uma generalização de uma tendência linear temporal que permite a inclinação da tendência da mudança gradual durante o período analisado e minimiza a soma dos desvios entre a tendência e a série atual. Ou seja,

O filtro HP é a generalização de uma tendência linear do tempo que permite que a inclinação da tendência mude gradualmente ao longo do tempo. Formalmente, o filtro HP minimiza a soma dos desvios quadrados entre a tendência e a série atual, mantendo a tendência suavizada. Se não houvesse penalidades, o filtro produziria a série original, se a pena fosse muito alta, resultaria em tendência linear (Ball; Mankiw, 2002, p.122).

Uma vez alcançadas as séries necessárias para a equação de transferência, ou seja, expectativa de inflação, a taxa de inflação (INPC) e a taxa de desemprego suavizada, no próximo subitem se verifica a estimação desta equação.

\subsection{Resultados empíricos para a NAIRU da economia brasileira}

Como ambas as séries se mostraram estacionárias ${ }^{10}$ a equação (2) deve apresentar a formulação da equação (5) nas estimações.

7 Para tal objetivo, também é necessária que a variável explicativa seja exógena. No Anexo II deste trabalho é verificado o teste de endogeneidade de Hausman, o qual com $1 \%$ de significância confirma esta hipótese.

8 Vale ressaltar que a série é altamente sazonal (por exemplo, é menor no último trimestre por conta dos empregos temporários). Dessa forma, antes de suavizada ela foi dessazonalizada pelo método multiplicativo X12 ARIMA.

9 Várias foram as metodologias utilizadas para a extração da tendência da série desemprego. Inclusive a decomposição da série em tendência, sazonalidade, ciclos e componente irregular com base no método da máxima verossimilhança através do filtro de Kalman (pacote Stamp 5.0). As estimativas, porém, mostraram-se mais significativas pelo filtro HP.

10 A parir do teste de Augmented Dickey-Fuller (ADF) para as séries da inflação e para a taxa de desemprego, como ressalva Portugal e Madalozzo (2000), por se tratar de uma série limitada, deve-se transformar a série para logit a fim de utilizar o teste ADF para raiz unitária. Uma vez feito isso, a série se mostrou estacionária. 


$$
\pi_{t}-\pi_{t}^{e}=\alpha+A(L)\left[\pi_{t}-\pi_{t}^{e}\right]+C(L) U_{t}+B(L) \varepsilon_{t}
$$

Mesmo se tratando de um período mais estável da economia brasileira, as séries mostraram certa variabilidade principalmente no ano de $2002^{11}$ por isso, dummies ${ }^{12}$ foram testadas e a d2002 correspondente ao ano de 2002 se mostrou significativa nas estimações. Deste modo, a equação (5) toma a seguinte forma

$$
\pi_{t}-\pi_{t}^{e}=\alpha+A(L)\left[\pi_{t}-\pi_{t}^{e}\right]+C(L) U_{t}+\delta d 2002+e_{t}
$$

Onde: $\quad$ d2002 = Dummy correspondente ao ano de 2002;

$$
e_{t}=\text { resíduos da estimativa. }
$$

Os resíduos da equação (6) não são necessariamente ruídos brancos. Esse fato pode ser proveniente do modelo a ser estimado, pois, a inflação pode ser explicada por mais variáveis do que apenas a taxa de desemprego, como é no caso da curva de Phillips. Por isto, a omissão de variáveis pode gerar resíduos que não se caracterizem por ruído branco. Logo, foi necessária a determinação do grau do polinômio $B(L)$ da equação (5), ou seja,

$$
e_{t}=B(L) \varepsilon_{t}
$$

O grau do polinômio é identificado pelas FAC e FACP obtido por um modelo ARMA. ${ }^{13} \mathrm{O}$ modelo mais adequado ao propósito deste artigo foi, mais uma vez, o processo autorregressivo de ordem um (AR(1)). Dessa forma, este foi incorporado às estimativas alcançadas, apresentadas na equação (8).

$$
\begin{array}{ccc}
\pi_{t}-\pi_{t}^{e}=0,221143+0,37883\left[\pi_{t-1}-\pi_{t-1}^{e}\right]-0,460844\left[\pi_{t-2}-\pi_{t-2}^{e}\right] \\
(0,63)^{14} & (2,16) & (-3,15)
\end{array}
$$

11 Ano que a economia brasileira esteve suscetível ao efeito Lula, a crise da Argentina e ao "apagão" energético, dentre outros.

12 Sabendo-se que em 2008 a região metropolitana de Fortaleza foi inserida ao cálculo da taxa de desemprego aberta da PED, testou-se uma dummy para este ano, porém, como explicitado no texto, apenas a do ano de 2002 foi significativa e efetivamente usada nas estimações. 13 Da mesma forma que a série expectativa de inflação foi gerada.

14 O coeficiente linear da curva de Philips estimada não se mostrou significativo. Porém, uma vez que sua utilização é necessária para o cálculo da taxa NAIRU, ele foi mantido na 


$$
\begin{aligned}
& +0,718209 U_{t}-0,733385 U_{t-2}+0,381529 d 2002-0,354540 \varepsilon_{t-1} \\
& (1,84) \quad(-1,83) \quad(2,15) \quad(-1,77)^{15} \\
& R^{2}=0,369822 \quad D W=2,0322229
\end{aligned}
$$$$
\text { AR } 1 \rightarrow \text { Prob }=0,2857
$$

$$
A R C H 1 \rightarrow \text { Prob }=0,8426
$$

A equação (8) foi estimada a partir do método das variáveis instrumentais (IV). Foi necessário o uso desse método pelo fato de haver correlação entre a variável endógena e o termo de erro (Bueno, 2011). Dos possíveis motivos que caracterizam a necessidade do uso de IV, o modelo estimado se enquadra no caso de variáveis omitidas e regressão dinâmica.

A vantagem de usar IV para a estimação é a possibilidade do uso de instrumentos adicionais, isto porque, como o objetivo do trabalho é testar uma forma funcional específica (Curva de Phillips), fica-se limitado a apenas duas variáveis, ou seja, inflação e desemprego. Sob este prisma, é necessário encontrar variáveis que sejam correlacionadas com a variável endógena (taxa de inflação) e não correlacionadas com o erro ou com a variável exógena (taxa de desemprego) para serem usadas como instrumentos. ${ }^{16}$

A manipulação algébrica da equação (8) possibilitará a estimação da taxa NAIRU para o Brasil. Assumindo que, segundo Portugal e Madalozzo (2000), a NAIRU é o valor do desemprego quando as expectativas da inflação se igualam a inflação corrente e, isolando a taxa de desemprego, alcançamos a equação (9).

$$
N A I R U=U_{t}^{*}=\frac{0,221143+0,381529 d 2002-0,354540 \varepsilon_{t-1}}{0,733385-0,718209}
$$

O Gráfico 2 apresenta os valores da taxa de desemprego e a NAIRU estimada para cada trimestre do período em questão, de 2001 ao segundo trimestre de 2013.

especificação do modelo.

15 Entre parênteses a estatística $t$ calculada.

16 Os instrumentos utilizados foram: Taxa de câmbio, Taxa Selic e crescimento do Produto Interno Bruto (PIB), todos disponibilizados pelo IPEA. 


\section{Gráfico 2 Taxa de desemprego DIEESE e NAIRU para o Brasil}

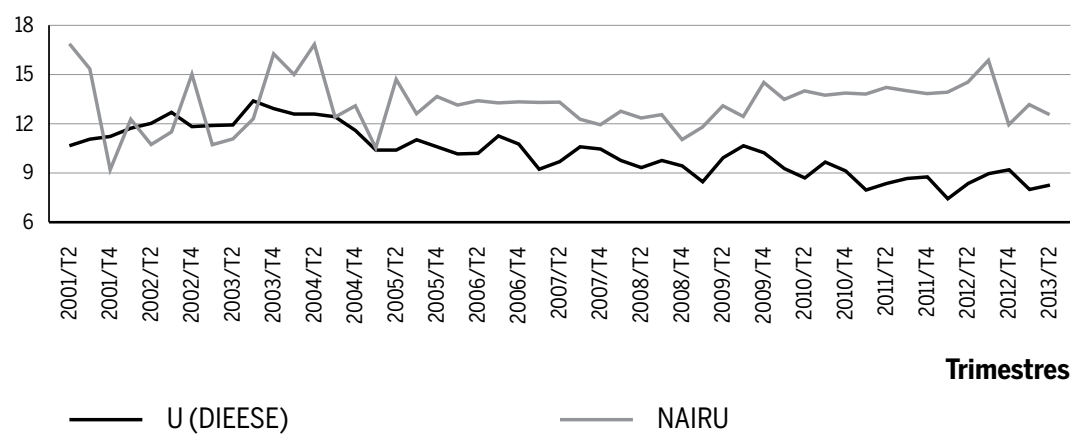

Fonte: Instituto Brasileiro de Geografia e Estatística (IBGE), elaboração própria.

A relação esperada acontece à medida que a NAIRU supera a taxa de desemprego ocasionando uma aceleração da taxa de inflação. Para testar esta relação rodamos uma regressão simples relacionando as variáveis em questão - a diferença entre a taxa de desemprego e a NAIRU e a inflação corrente (INPC), representada pela equação (10).

$$
\begin{aligned}
U_{t}-\operatorname{NAIRU}_{t}= & 1,961 \pi_{t} \\
& (2,656)^{17}
\end{aligned}
$$

Onde: $U_{t}=$ taxa de desemprego no período $t$;

$N A I R U_{t}=$ NAIRU estimada para o período $t$;

$\pi_{t}=$ inflação para o período $t$.

A obtenção do coeficiente positivo poderia indicar que a taxa NAIRU alcançada não é consistente para a economia brasileira, pois, a relação esperada pela relação de Phillips seria negativa. Entretanto, quando se analisa a Figura A1, que relaciona a taxa de desemprego e a taxa de inflação com as suas respectivas tendências um indicativo da robustez da estimativa alcançada é sinalizado.

No Gráfico 3 percebe-se que depois da estabilização da economia brasileira, existe uma reversão da relação entre as taxas que caracterizam a curva de Phillips. No primeiro gráfico, pode ser observado o período até a adoção do regime de metas de inflação e, neste período, a relação negativa 
entre inflação e desemprego se dá de forma clara. Contudo, após a implementação do regime de metas, a taxa de desemprego instala tendência de queda e a taxa de inflação permanece praticamente estável.

\section{Gráfico 3 Taxa de inflação (INPC), taxa de desemprego (U) e tendências}

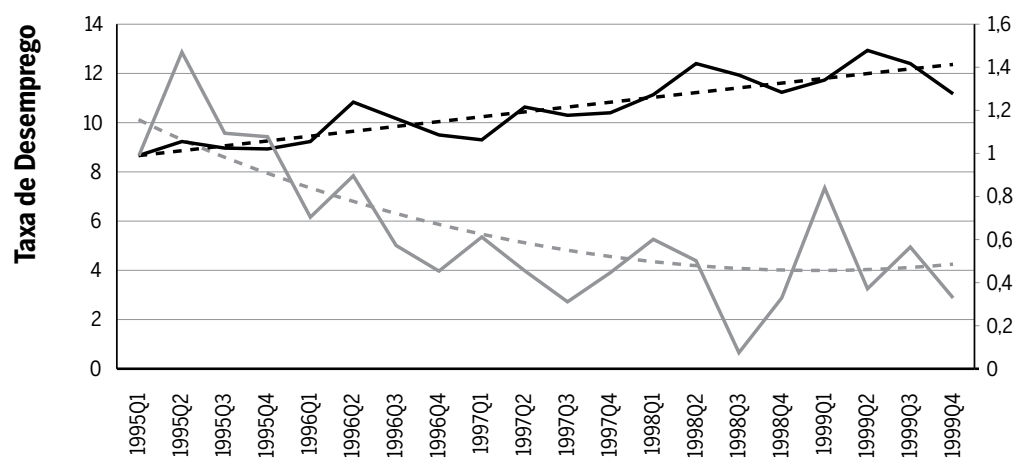

Trimestres
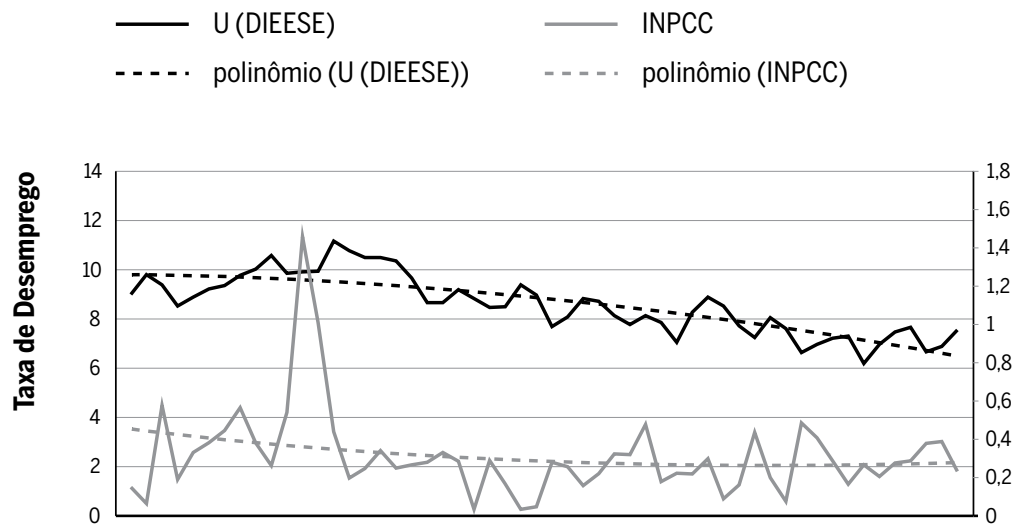

윯

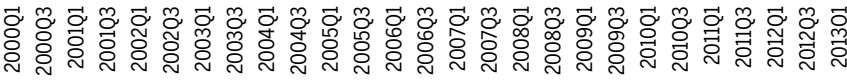

\section{Trimestres}

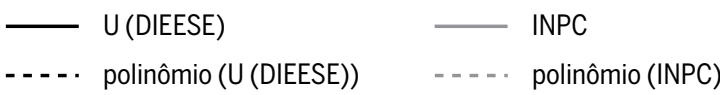

Fonte: Instituto Brasileiro de Geografia e Estatística (IBGE), elaboração própria.

Constatação de acordo com o trabalho de Silva Filho (2008), no qual o autor também encontrou relação positiva entre inflação e desemprego. 
Dados brasileiros trazem a complicação adicional de que não houve nenhum trade-off entre inflação e desemprego desde a estabilização. Pelo contrário, a correlação foi positiva. Esta evidência aponta fortemente para a importância dos choques de oferta sobre a dinâmica da inflação recentes no Brasil (Silva Filho, 2008, p. 26, tradução nossa).

Além disso, uma possível explicação para taxa NAIRU permanecer acima da taxa de desemprego como no Gráfico 2 pode estar relacionado a produtividade ${ }^{18}$ da economia brasileira estar em um processo de decréscimo (Gráfico 4).

\section{Gráfico 4 Taxa de inflação e produtividade da economia brasileira}

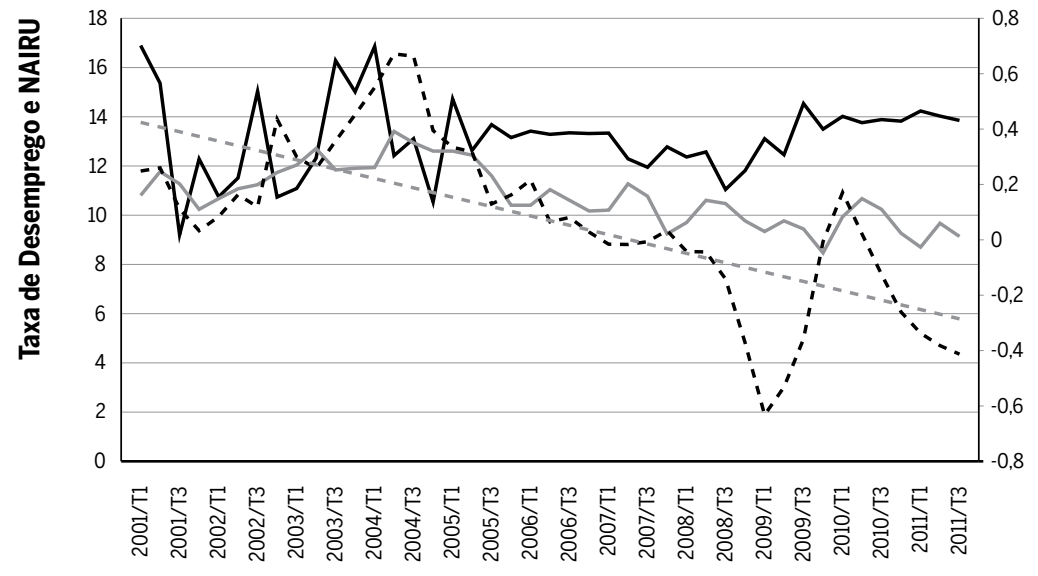

Fonte: Instituto Brasileiro de Geografia e Estatística (IBGE), elaboração própria.

Ball e Mankiw (2002) apontam que variações na NAIRU parecem estar relacionadas a flutuações na produtividade. Destacam ainda que na década de 1970 nos Estados Unidos, a NAIRU aumentou quando o crescimento da produtividade estava decaindo.

Por fim, outra forma de representar a Curva de Phillips é relacioná-la

18 A metodologia empregada no desenvolvimento da variável produtividade segue a usada por Souza Neto e Curado (2003) na qual se calcula a diferença entre os logaritmos da produção física da indústria (disponibilizada pela FIESP) e da série horas pagas na indústria geral. Vale ressaltar que as duas séries foram dessazonalizadas pelo método X12 ARIMA. 
com a Lei de Okun, ${ }^{19}$ o gráfico cinco mostra a relação entre o produto natural $^{20}$ e a taxa de inflação da economia brasileira.

O que se pode verificar é que, mesmo que sutil, a relação inversa no período de análise entre o produto natural e a taxa de inflação parece estar mais visível que quando esta taxa foi comparada a taxa de desemprego no Gráfico três. Sachsida, Ribeiro e Santos (2009) consideram, ao estimar uma Curva de Phillips para a economia brasileira, o hiato do produto, a utilização da capacidade instalada da indústria brasileira e a taxa de desemprego do estado São Paulo. ${ }^{21}$ Alcançam que o hiato e a capacidade instalada não são as melhores proxies para representar o custo marginal e que a taxa de desemprego forneceu os melhores resultados. Ou seja, a utilização da taxa de desemprego é consistente para estimar a Curva de Phillips da economia brasileira.

\section{Gráfico 5 Produto natural, taxa de inflação e respectivas tendências da economia brasileira}

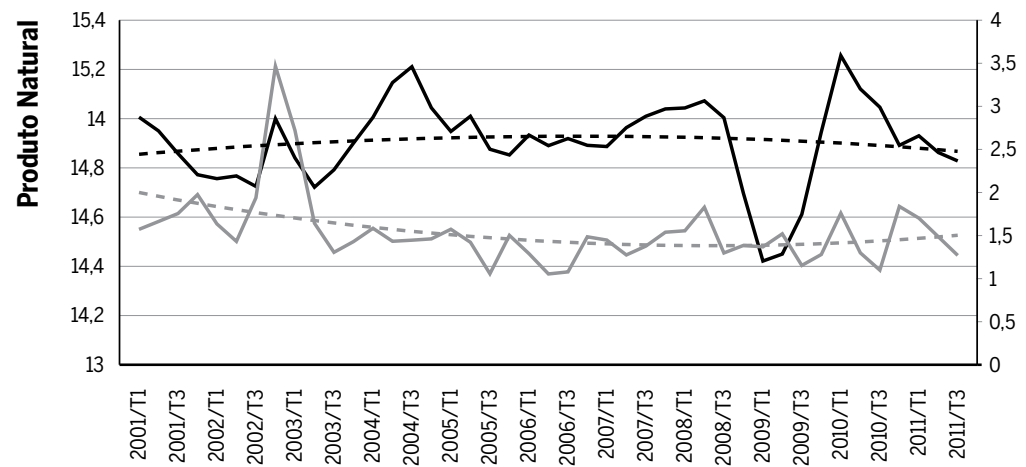

Fonte: Elaboração própria.

19 Segundo Souza Júnior (2005), a redução da taxa de desemprego está relacionada com o crescimento da diferença entre o produto natural e o efetivo (gap do produto). Relação esta que se apresenta na Lei de Okun, ou seja, podemos, ao invés de relacionar inflação e desemprego, analisar inflação e gap do produto e, assim, fazer inferências sobre o desemprego.

$20 \mathrm{O}$ produto natural foi alcançado um filtro multivariado utilizando a mesma metodologia que Areosa (2004). Com base em uma Cobb-Douglas, filtram-se simultaneamente os gaps do desemprego, da capacidade instalada e do produto e, com uma regressão simples de mínimos quadrados, alcança-se o produto natural. Ressaltando que os dados utilizados são disponibilizados pelo IBGE.

21 Mesma taxa utilizada por este artigo. 


\section{Considerações finais}

Este artigo se propôs a estimar a NAIRU para a economia brasileira com dados trimestrais de 2000 ao segundo trimestre de 2013, a partir de uma curva de Phillips. A metodologia empregada confronta a equação de transferência na qual a variável endógena, no caso a diferença entre a inflação corrente e a esperada, com valores correntes e defasados dela própria e da taxa de desemprego.

A primeira constatação verificada diz respeito ao fato da NAIRU ter permanecido na maior parte do período analisado em patamar superior a taxa de desemprego, o que poderia ou invalidar a significância das estimativas calculadas ou justificar uma aceleração da taxa de inflação, uma vez que a curva de Phillips mostra a relação negativa entre a inflação e a taxa de desemprego.

Entretanto, ao se fazer uma análise mais minuciosa desta relação, verifica-se que nos dados em nível a taxa de desemprego e de inflação apresentam tendências parecidas a partir dos anos 2000, levando a acreditar que a esta relação deveria ser incluída outras variáveis como a produtividade, que mostra a relação negativa esperada.

Dessa forma, as principais conclusões deste artigo dizem respeito à mudança do coeficiente de correlação entre a taxa de desemprego e a taxa de inflação, que passou de negativo para positivo e da permanência da taxa NAIRU acima da taxa de desemprego no período em questão. Considerando que a taxa de desemprego não aceleradora da inflação mostrou-se acima da taxa de desemprego, é interessante que medidas de elevação a produtividade da economia sejam tomadas, com o propósito do país poder apresentar uma taxa de desemprego cada vez mais baixa, sem que isso cause, como contrapartida, uma externalidade negativa em termos de aceleração do nível de preços.

Uma vez que este trabalho não esgota as possibilidades de estudo do tema, acredita-se que a investigação da reversão da relação deve ser levada em consideração para, assim, se solidificar esta constatação.

\section{Referências}

AREOSA, M. B. M. Combining Hodrick-Prescott filtering with a production-function approach to estimate the output gap. Texto apresentado no Seminário de 5 anos de Metas de Inflação, Banco Central do Brasil, 2004. 
BALL, L.; MANKIW, G. N. The NAIRU in theory and practice. Journal of Economic Perspectives. v. 16, n. 14, p. $115-136,2002$.

BRESSER-PEREIRA, L. C.; GOMES, C. O regime de metas de inflação no Brasil e a armadilha da taxa de juros/taxa de câmbio. In: OREIRO, J. L.; PAULA, L. F.; SOBREIRA, R. (Orgs.) Política monetária, bancos centrais e metas de inflação: teoria e experiência brasileira. Rio de Janeiro: FGV, 2009. p. 21-51.

BUENO, R. de L. da S. Econometria de Séries Temporais. 2a Ed. Cegage Learning, 2011.

FERREIRA, A.; ESTEVES, P. S.; FÉLIX, R. M. Estimates of the NAIRU for Brazil using the Ball-Mankiw approach. Belo Horizonte, 2003. (Notas Técnicas do CIPE)

FLORI, P. M. Desemprego de Jovens: um estudo sobre a dinâmica do mercado de trabalho juvenil brasileiro. Dissertação (Mestrado em Economia) - Faculdade de Economia, Administração e Contabilidade, USP, São Paulo, 2003.

FRIEDMAN, M.. The Role of Monetary Policy. The American Economic Review, v. 58, p. 1-17, 1968.

GIAMBIAGI, F.; CASTRO, L. B. de; HERMAN, J. (Org.). Economia Brasileira Contemporânea. 9a ed. Rio de Janeiro: Campus, 2005.

GORDON, R. J. The time-varying NAIRU and its implications for economics policy. Journal of Economic Perspective, v. 11, n. 1, 1997.

GREMAUD, A. P.; VASCONCELLOS, M. A. S.; TONETO JÚNIOR, R. Economia Brasileira Contemporânea. 7a. ed. São Paulo: Atlas, 2009.

LIMA, R. C. S. et al. A curva de Phillips e sua aplicação na Economia Contemporânea. 2000. Disponível em: <http://www.pet-economia.ufpr.br/banco_de_arquivos/00002_Rodrigo_cl_ lima_e_silva_-_curva_de_philips_e_aplicacao.pdf>. Acesso em: 17 set. 2013.

MENDONÇA, H. F. Mecanismos de transmissão monetária e a determinação da taxa de juros: uma aplicação da regra de Taylor ao caso brasileiro. Economia e Sociedade, Unicamp, jun. 2001.

PHELPS, E. S.; TAYLOR, J. B.. Stabilizing Powers of Monetary Policy under Rational Expectations. Journal of Political Economy, v.85, p. 163-190, 1977.

PICHETTI, P.; CHAHAD, J. P. A evolução da taxa de desemprego estrutural do Brasil: uma análise entre regiões e características dos trabalhadores. In: ENCONTRO NACIONAL DA ASSOCIAÇÃO NACIONAL DE PÓS-GRADUAÇÃO EM ECONOMIA - ANPEC, Salvador, 2003.

PORTUGAL, M. S.; MADALOZZO, R. C. Um modelo de NAIRU para o Brasil. Revista de Economia Política, n. 4, p. 26-47, 2000.

SACHSIDA, A., RIBEIRO, M., SANTOS, C. H. A curva de Phillips e a experiência brasileira. IPEA - Texto para Discussão no 1.429, Rio de Janeiro, out. 2009.

SILVA FILHO, T. N. T. Searching for the NAIRU in a large relative price shock's economy Brazilian case. BACEN. Working Paper Series n.163, 2008.

SOUZA JÚNIOR, J. R. Produto potencial: conceitos, métodos de estimação e aplicação à economia brasileira. IPEA - Texto para Discussão, n. 1.130, Rio de Janeiro, nov. 2005.

SOUZA NETTO, C. R. de; CURADO, M. L. Produtividade do trabalho, salários reais e desemprego na indústria de transformação do Brasil na década de 90. ENCONTRO NACIONAL DE ECONOMIA - ANPEC, Porto Seguro, 2003. 


\section{Sobre os autores}

Luma de Oliveira - luma_de_oliveira@hotmail.com Universidade Federal do Rio Grande do Sul, Porto Alegre, RS.

Marcelo SavinoPortugal - msp@ufrgs.br

Universidade Federal do Rio Grande do Sul, Porto Alegre, RS.

Mateus Boldrine Abrita - mateusabrita@hotmail.com

Universidade Federal do Rio Grande do Sul, Porto Alegre, RS/UEMS, Campo Grande, MS.

\section{Sobre o artigo}

Recebido em 25 de agosto de 2014. Aprovado em 14 de abril de 2015. 


\section{ANEXO I}

Especificação do modelo ARIMA para a taxa de inflação esperada.

Figura A1 Correlograma da série taxa de inflação

\begin{tabular}{|c|c|c|c|c|c|c|c|c|}
\hline \multicolumn{2}{|c|}{ Autocorrelação } & \multicolumn{2}{|c|}{$\begin{array}{l}\text { Correlação } \\
\text { Parcial }\end{array}$} & & AC & PAC & Q-Stat. & Prob. \\
\hline 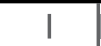 & $\bar{T}$ & I & I & 1 & 0.465 & 0.465 & 12.361 & 0.000 \\
\hline I & I & I & I & 2 & 0.042 & -0.223 & 12.464 & 0.002 \\
\hline I & I & I & 1 & 3 & 0.058 & 0.186 & 12.665 & 0.005 \\
\hline I & ال & I & I & 4 & 0.190 & 0.114 & 14.855 & 0.005 \\
\hline I & 1 & I & I & 5 & 0.140 & -0.019 & 16.065 & 0.007 \\
\hline I & I & I & I & 6 & 0.020 & -0.016 & 16.089 & 0.013 \\
\hline I & I & I & I & 7 & 0.010 & 0.025 & 16.095 & 0.024 \\
\hline I & I & I & I & 8 & 0.011 & -0.052 & 16.103 & 0.041 \\
\hline I & I & I & I & 9 & 0.050 & 0.071 & 16.270 & 0.061 \\
\hline 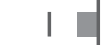 & I & I & I & 10 & -0.113 & -0.236 & 17.144 & 0.071 \\
\hline I & I & I & I & 11 & -0.210 & -0.051 & 20.250 & 0.042 \\
\hline I & I & I & I & 12 & -0.052 & 0.103 & 20.447 & 0.059 \\
\hline I & I & I & I & 13 & -0.089 & -0.241 & 21.028 & 0.072 \\
\hline 1 & I & 1 & I & 14 & -0.170 & 0.029 & 23.225 & 0.057 \\
\hline I & I & I & I & 15 & -0.159 & -0.016 & 25.193 & 0.047 \\
\hline I & I & I & I & 16 & -0.010 & 0.057 & 25.200 & 0.066 \\
\hline I & I & I & I & 17 & -0.038 & -0.071 & 25.317 & 0.088 \\
\hline 1 당 & I & I & I & 18 & -0.136 & -0.056 & 26.875 & 0.081 \\
\hline 1 & I & I & I & 19 & -0.073 & 0.085 & 27.339 & 0.097 \\
\hline I & I & I & I & 20 & 0.014 & 0.021 & 27.356 & 0.126 \\
\hline I & I & I & I & 21 & 0.081 & 0.002 & 27.962 & 0.141 \\
\hline I & I & I & I & 22 & 0.037 & 0.046 & 28.090 & 0.173 \\
\hline 1 & I & I & I & 23 & -0.060 & -0.102 & 28.438 & 0.200 \\
\hline I & I & I & I & 24 & -0.022 & 0.015 & 28.488 & 0.240 \\
\hline
\end{tabular}

Fonte: Elaboração própria com base no software Eviews.

Nota-se que a função de autocorrelação (FAC) decrescente com oscilação e que a função de autocorrelação parcial (FACP) apresenta o pico na defasagem um. Fatos estes que caracterizam um modelo autorregressivo de ordem um $(\mathrm{AR}(1))$. 
Figura A2 Modelo AR(1) para a taxa de inflação

\begin{tabular}{lrrrrr}
\hline Variável & Coeficiente & Erro Padrão & Estatística $\boldsymbol{t}$ & Prob. \\
\hline C & 1.538733 & 0.089757 & 17.14334 & 0.0000 \\
\hline AR (1) & 0.466372 & 0.123190 & 3.785795 & 0.0004 \\
\hline R2 & 0.219375 & Média da variável dependente & 1.536098 \\
\hline R2 ajustado & 0.204068 & $\begin{array}{l}\text { Desvio Padrão da variável } \\
\text { dependente }\end{array}$ & 0.390806 \\
\hline Erro padrão da regressão & 0.348657 & $\begin{array}{l}\text { Critério de informação } \\
\text { de Akaike }\end{array}$ & 0.767551 \\
\hline $\begin{array}{l}\text { Soma dos quadrados } \\
\text { dos resíduos }\end{array}$ & 6.199660 & Critério de Schwarz & 0.841902 \\
\hline Log verossimilhança & -18.34011 & Critério Hannan-Quinn & 0.796143 \\
\hline F-statistic & 14.33224 & Estatística de Durbin-Watson & 1.794660 \\
\hline Prob (estatística F) & 0.000405 & & \\
\hline Raízes AR invertidas & .47 & & \\
\hline
\end{tabular}

Fonte: Elaboração própria com base no software Eviews.

O que se verifica na Figura A2 é a regressão que gerou a série prevista para a taxa de inflação esperada. Cabe destacar que o modelo se mostrou significativo e que o teste $t$ para comprovar a significância do processo autorregressivo é altamente significativo. 


\section{ANEXO II}

Teste de endogeneidade para a série Taxa de Desemprego

\section{Teste de Hausman}

Para testar se a série taxa de desemprego é exógena a variável explicada, ou seja, a diferença entre a taxa de inflação corrente e esperada, efetuou-se o teste de Hausman. Esse teste consiste na estimação do modelo por variáveis instrumentais (equação 8) usar os valores previstos da variável endógena em uma regressão simples de mínimos quadrados. A hipótese nula de exogeneidade é aceita quando o coeficiente dos valores previstos não é significativamente diferente de zero. Desta forma, sendo que a estatística t deste teste foi de 0,182623 , comprova-se a não significância do coeficiente já a $1 \%$. 